\title{
Observation of algebraic time order for two-dimensional dipolar excitons
}

\author{
Suzanne Dang, ${ }^{1}$ Marta Zamorano, ${ }^{1}$ Stephan Suffit $\odot,{ }^{2}$ Kenneth West, ${ }^{3}$ Kirk Baldwin, ${ }^{3}$ \\ Loren Pfeiffer, ${ }^{3}$ Markus Holzmann, ${ }^{4}$ and François Dubin ${ }^{1}$ \\ ${ }^{1}$ Institut des Nanosciences de Paris, CNRS and Sorbonne Université, 4 place Jussieu, 75005 Paris, France \\ ${ }^{2}$ Laboratoire de Materiaux et Phenomenes Quantiques, Université Paris Diderot, 75013 Paris, France \\ ${ }^{3}$ PRISM, Princeton Institute for the Science and Technology of Materials, Princeton University, Princeton, New Jersey 08540, USA \\ ${ }^{4}$ Université Grenoble Alpes, CNRS, LPMMC, 3800 Grenoble, France
}

(Received 30 January 2020; revised 10 June 2020; accepted 18 June 2020; published 13 July 2020)

\begin{abstract}
We study dipolar excitons confined in a flat trap of a GaAs bilayer heterostructure. By quantifying density and density fluctuations at sub-Kelvin temperatures, we unveil the excitons' universal equation of state. This allows us to infer thermodynamically the critical phase-space density for the transition expected between normal and superfluid regimes. The region of this crossover is directly confirmed by a net change in the excitons' temporal coherence, from an exponential to an algebraic decay with an exponent compatible with the Berezinskii-Kosterlitz-Thouless theory.
\end{abstract}

DOI: 10.1103/PhysRevResearch.2.032013

Dipolar quantum gases constitute a versatile playground to explore exotic collective phenomena emerging in a strong interaction regime [1]. In two dimensions, important correlation effects are predicted for moderate and high densities [2], causing substantial deviations from dilute gas behavior. Very recently, supersolid properties [3-5] have been reported for ultracold atoms with a large permanent magnetic dipole. In semiconductors, even stronger dipolar interactions are accessible, using bilayer heterostructures where electrons and holes are spatially separated but bound by a Coulomb attraction [6]. Dipolar excitons are thus formed, characterized by a giant and well-oriented electric dipole moment perpendicular to the bilayer [7].

Recent experiments have reported that dipolar excitons can realize a two-dimensional quantum gas $[7,8]$ for which quasilong-range coherence $[9,10]$, quantized vortices $[10,11]$ have been reported in GaAs bilayers. The excitons' quasicondensation must then occur following the Berezinskii-KosterlitzThouless (BKT) mechanism [12,13], since for this reduced dimensionality interactions prevent Bose-Einstein condensation with true long-range order, at any finite temperature [14]. Accordingly, the quasicondensation crossover is characterized by the emergence of algebraic quasi-long-range order which constitutes a key feature of two-dimensional superfluid phase transitions, notoriously difficult to observe experimentally. Indeed, so far it has only been reported for ultracold atomic gases $[15,16]$ and driven-dissipative exciton polaritons [17], which are both characterized by weak interactions so that the BKT transition competes with Bose-Einstein condensation in trapped geometries $[18,19]$.

Published by the American Physical Society under the terms of the Creative Commons Attribution 4.0 International license. Further distribution of this work must maintain attribution to the author(s) and the published article's title, journal citation, and DOI.
In this Rapid Communication, we first quantify thermodynamically the quasicondensate crossover region of twodimensional dipolar excitons confined in a flat trap of a GaAs bilayer. For that, we experimentally determine the equation of state and density fluctuations. Whereas an apparent scale invariance for these observables has been measured with ultracold atoms, even away from criticality [20-22], we report a net violation of scale invariance. Unlike in recent studies [11], it is resolved here owing to very precise measurements of the density profiles. Thus, we confirm the strongly interacting character of the excitons' dipolar interactions. Also, we show that a universal behavior $[22,23]$ is restored within a large fluctuating region around the quasicondensate crossover. Importantly, close to the transition we observe a clear change in the excitons' temporal phase coherence, from an exponential to an algebraic decay, revealing the buildup of a quasilong-range ordered phase. The exponent of the algebraic decay $\eta$ decreases with increasing phase-space density, and is compatible with $\eta_{c} \simeq 0.25$ predicted by the BKT theory $[12,13,24]$ at criticality. This observation provides a direct signature [25] of a Berezinskii-Kosterlitz-Thouless scenario for the quasicondensation of dipolar excitons.

Our experiments start by injecting optically dipolar excitons in a $20-\mu \mathrm{m}$-wide trap of an ultrapure GaAs double quantum well. This excitation lasts $100 \mathrm{~ns}$, repeated at a frequency of $1.5 \mathrm{MHz}$, while at a variable delay after termination of the optical loading we analyze the photoluminescence reemitted by the trapped gas and which reflects directly the fundamental properties of the excitonic cloud [6,7]. Indeed, the energy of the photoluminescence $E_{X}$ provides the density in the trap $n(\mathbf{r})$ since $E_{X}$ scales as $\left[E_{t}(\mathbf{r})+u_{0} n(\mathbf{r})\right]$, the second term reflects the strength of the repulsive dipolar interactions between excitons [26-28], and the first term the profile of the trapping potential. Additional information is gained from the photoluminescence time coherence, which characterizes the collisional dynamics and thus quantifies the excitons' temporal phase coherence. 

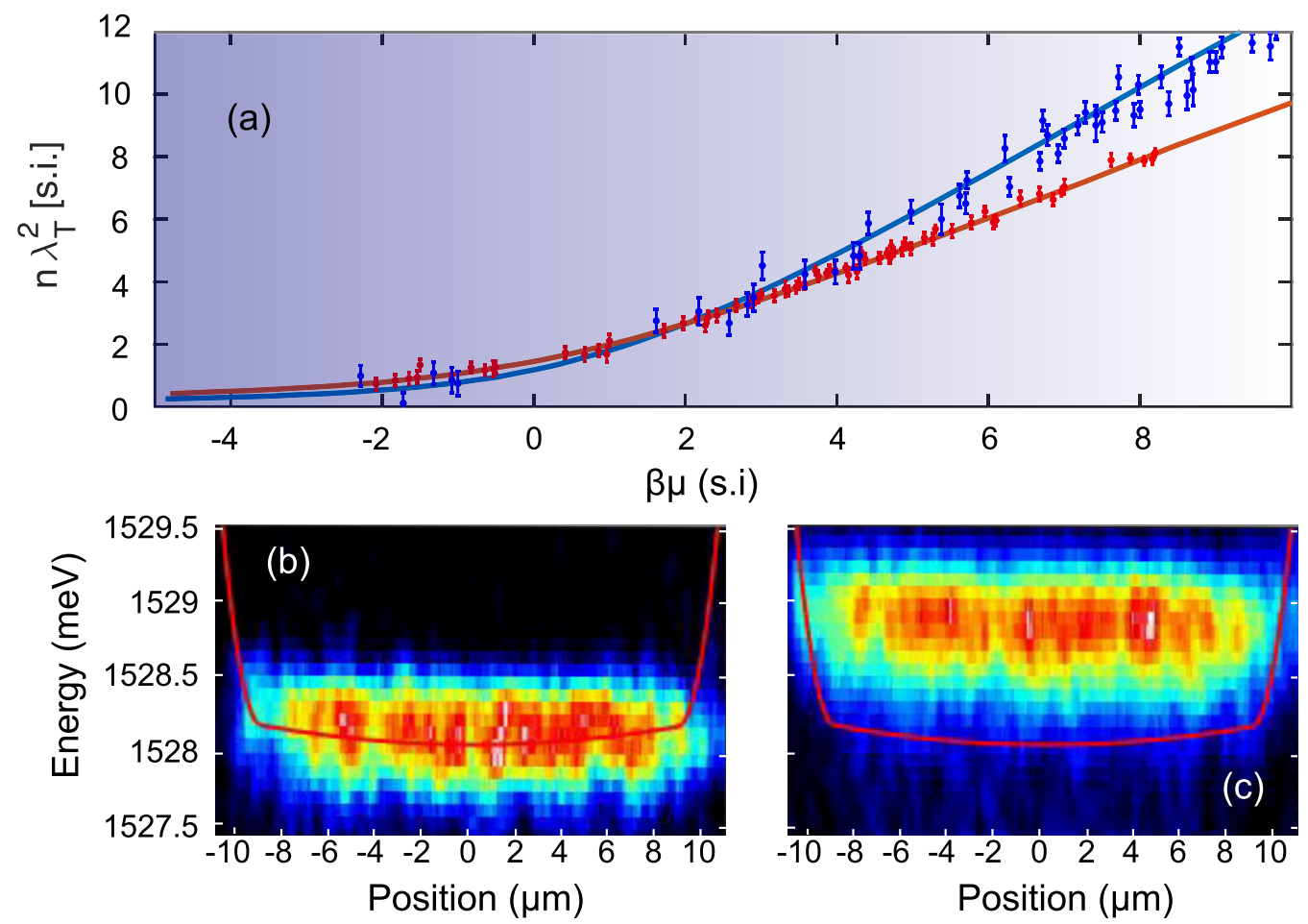

FIG. 1. (a) Phase-space density $D=n \lambda_{T}^{2}$ measured at $T=340 \mathrm{mK}$ (blue) and $750 \mathrm{mK}$ (red), together with the results of Monte Carlo calculations for $\tilde{g}=4$ and 6 , respectively (solid lines). $D$ is obtained by analyzing the profile of the photoluminescence energy across the trap from a statistical ensemble of 20 repetitions for every experimental condition. Experimentally, the density in the trap is varied by scanning the delay to the loading phase. It is (c) about $2 \times 10^{10} \mathrm{~cm}^{-2}$ at the center when the delay is set to $150 \mathrm{~ns}$ and (b) around $10^{9} \mathrm{~cm}^{-2}$ when it is set to $370 \mathrm{~ns}$. In the latter case the photoluminescence energy reproduces the profile of the trapping potential fitted by the solid red line. Error bars display the statistical deviations of our measurements.

In our system, excitons are characterized by a dipole moment $d$, oriented perpendicular to the GaAs bilayer, with a magnitude controlled by the spatial separation between the two quantum wells. The effective exciton-exciton interaction potential is well described [26-28] by $\mathrm{V}_{\text {eff }}(\mathbf{r})=\left(d_{\text {eff }}\right)^{2} /|\mathbf{r}|^{3}$, where $d_{\text {eff }}=d \sqrt{f}$ contains a screening amplitude $f$ that weakly depends on the exciton density in the dilute regime ( $f$ being of the order of 0.2 for $n \sim 10^{10} \mathrm{~cm}^{-2}$ at low temperatures) and may be adapted to also include correlation effects at intermediate and high densities [2]. In two dimensions, the dipolar potential $\mathrm{V}_{\text {eff }}$ is still sufficiently short ranged, so that asymptotic scattering properties at large distances are characterized by a dimensionless number $\tilde{g}[29,30]$.

In Fig. 1(a), we show the phase-space densities $D(\beta \mu)=$ $n \lambda_{\mathrm{T}}^{2}$ as a function of $\beta \mu=\mu / k_{\mathrm{B}} T, \lambda_{\mathrm{T}}=h / \sqrt{2 \pi m k_{\mathrm{B}} T}$ being the de Broglie thermal wavelength, $m$ the exciton mass, $T$ the temperature, and $\mu$ the chemical potential. These measurements are obtained within the local density approximation, i.e., by associating a chemical potential $\mu(\mathbf{r})=\mu_{0}-E_{t}(\mathbf{r})$ to the local exciton density $n(\mathbf{r})$ [11]. We measure the latter by first fitting the profile of the trapping potential from the spatially resolved photoluminescence long after ( $370 \mathrm{~ns}$ ) extinction of the loading pulse, i.e., when the density in the trap is vanishingly small [red line in Fig. 1(b)]. Then, the fitted profile is subtracted to the spatially resolved photoluminescence energy measured for shorter delays [Fig. 1(c)], so that we deduce the photoluminescence blueshift and therefore the excitons' density profile in the trap $n(\mathbf{r})$, computed by statistically averaging 20 realizations. Let us note that the concentration at the center of the trap is here set by the delay to the loading pulse. Varying this delay we explored the range of phase-space densities reported in Fig. 1(a), assigning the bath temperature to dipolar excitons. This assumption is supported by the temperature dependence of the photoluminescence spectrum [31].

Unlike quasi-two-dimensional atomic gases probed in the weak interaction regime [20-22], a net violation of scale invariance in our system is directly evidenced by comparing experiments at $T=340$ and $750 \mathrm{mK}$. Indeed, $D$ does not obey a unique function of $\beta \mu$. Instead, the distinct slopes observed between these two temperatures when $\beta \mu \gtrsim 0$ show that $\tilde{g}$ decreases with temperature, since $D(\beta \mu) \sim \beta \mu / \tilde{g}$ is expected in the Thomas-Fermi limit of a quasicondensate. A comparison with classical field Monte Carlo calculations taking into account the exciton's composite nature with four internal spin degrees of freedom $[11,18]$ indicates that $\tilde{g}$ increases from about 4 to 6 between 340 and $750 \mathrm{mK}$. Moreover, note that unlike Ref. [11], here, we directly observe the violation of scale invariance owing to a strong reduction of electrostatic fluctuations so that the exciton density is extracted with a high precision [31].

To further quantify the temperature dependence of $\tilde{g}$, we studied the exciton density variance $\sigma^{2}(\mathbf{r})$ that provides an independent and direct measure of $\tilde{g}$ in the quasicondensed regime [22]. Figure 2(a) shows the scaled variance $\sigma^{2} \lambda_{T}^{2}$ per $\mu \mathrm{m}^{2}$ at variable temperatures, within the hydrodynamic 

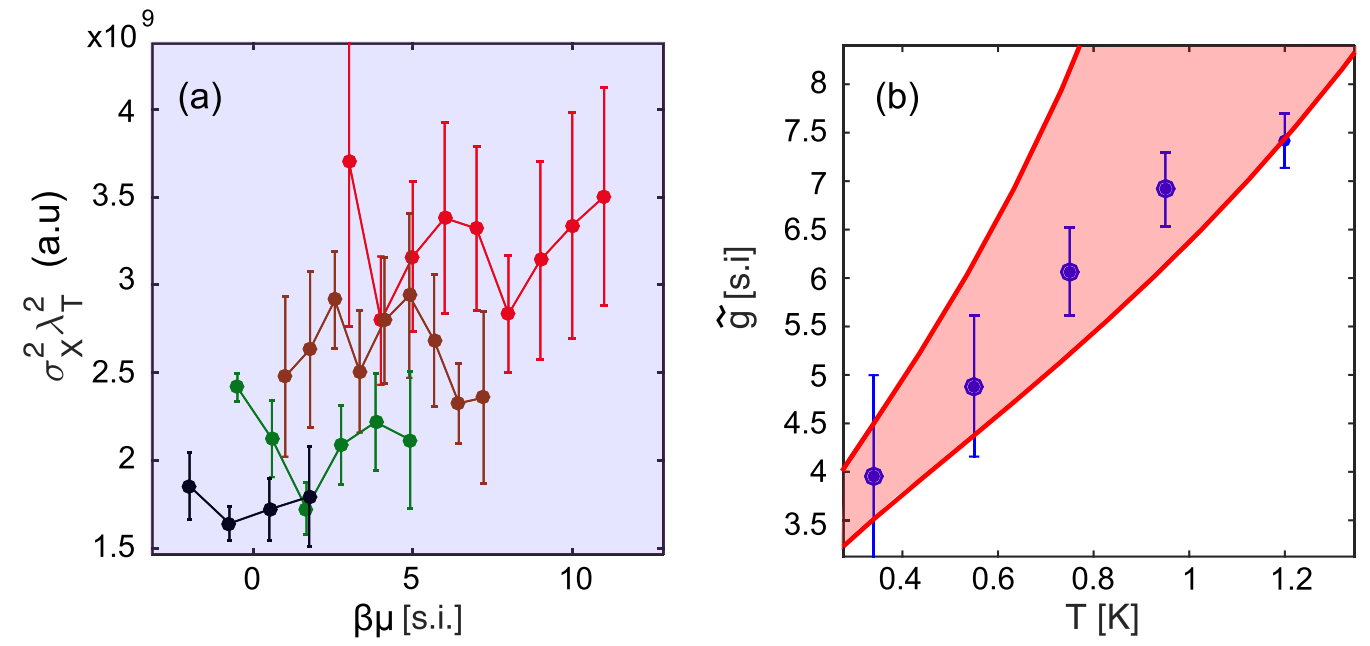

FIG. 2. (a) Rescaled density fluctuations $\sigma^{2} \lambda_{T}^{2}$ per $\mu \mathrm{m}^{2}$ measured at $340 \mathrm{mK}$ (red), $550 \mathrm{mK}$ (brown), $950 \mathrm{mK}$ (green), and $1.2 \mathrm{~K}$ (black). Data points are obtained by evaluating the variance for a set of 20 repetitions for every experimental setting, and $\sigma^{2} \lambda_{T}^{2}$ is then computed in intervals $\beta \mu \sim 1$ to reach higher precision. (b) Temperature scaling of $\tilde{g}$ extracted by averaging the data shown in (a) for each bath temperature (blue). The red shaded area marks the logarithmic scaling of $\tilde{g}$ with temperature expected for thermal two-body collisions with the effective dipolar exciton-exciton interaction (see Supplemental Material of Ref. [11]).
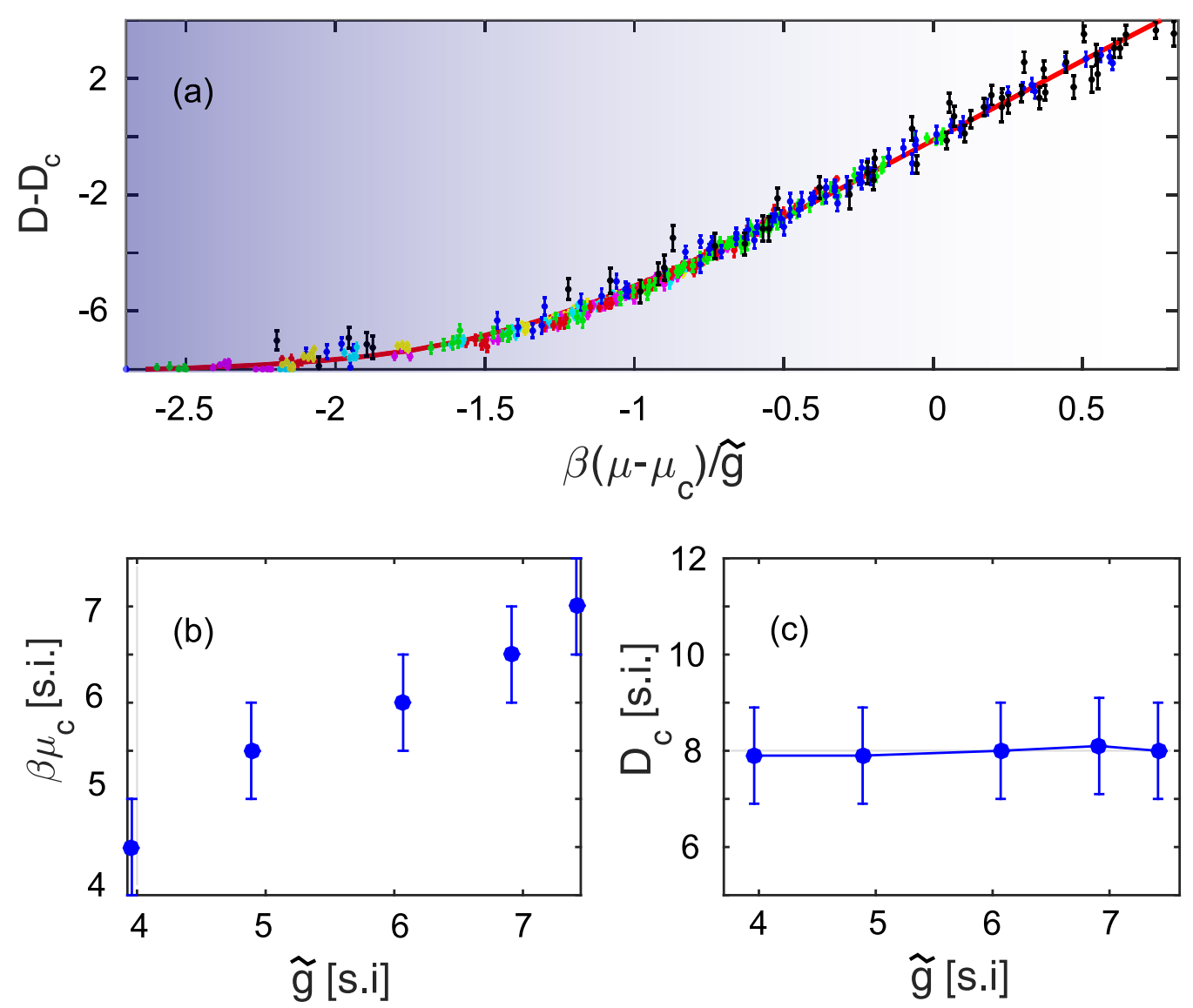

FIG. 3. (a) Equations of state $\left(D-D_{c}\right)$ in rescaled units $\beta\left(\mu-\mu_{c}\right) / \tilde{g}$, for experiments realized at $340 \mathrm{mK}, 550 \mathrm{mK}, 750 \mathrm{mK}, 950 \mathrm{mK}$, $1.2 \mathrm{~K}$, and $1.5 \mathrm{~K}$ (black, blue, green, red, cyan, and magenta, respectively). The solid red line displays the scaling predicted by Monte Carlo calculations. The resulting values of the critical chemical potential $\beta \mu_{c}$ and of the critical phase-space density $D_{c}$ are shown in (b) and (c), respectively. 
regime where $D$ scales linearly with $\beta \mu$. We note that $\sigma^{2} \lambda_{\mathrm{T}}^{2}$ weakly varies with $\beta \mu$ and approaches a mean value which mostly depends on $T$. Let us then underline that our experiments rely on cumulative averaging for each experimental settings. The measured local variance $\sigma^{2}(\mathbf{r})$ then corresponds to the mean-square deviation of the total number of excitons at a considered position $\mathbf{r}$. Furthermore, our optical resolution $(\sim 1 \mu \mathrm{m})$ largely exceeds the mean-field coherence length $(2 \tilde{g} n)^{-1 / 2}(\sim 25 \mathrm{~nm})$. We then determine the fluctuations of the local number of excitons so that $\sigma^{2} \lambda_{T}^{2}$ scales as $1 / \tilde{g}$ in the quasicondensed regime where $D$ scales linearly with $\beta \mu$ [31].

Averaging for each temperature $T$ the rescaled variance shown in Fig. 2(a), we deduce the dependence of $\tilde{g}$ as a function of $T$, imposing $\tilde{g}=4$ at $340 \mathrm{mK}$ according to the analysis of the equation of state. Figure 2(b) displays the thus obtained values, which are consistent with those from the Thomas-Fermi behavior used for the equation of state (Fig. 1), as expected from thermodynamics. Importantly, the temperature scaling of $\tilde{g}$ is consistent with a logarithmic dependence characteristic of two-dimensional scattering processes.

Despite a broken scale invariance, scaling behaviors may be retrieved by the universal variations expected close to a continuous phase transition such as the BKT transition. In particular, from the $\phi^{4}$ theory in two dimensions $[23,32]$, we expect the phase-space density to show a universal scaling, $\left(D-D_{c}\right)=F\left(\beta\left(\mu-\mu_{c}\right) / \tilde{g}\right)$ inside the fluctuating region, $\beta\left|\mu-\mu_{c}\right|<\tilde{g}$. Here, $F$ is a dimensionless function while $D_{c}$ and $\mu_{c}$ are the critical density and chemical potential which remove nonuniversal contributions with regular (smooth) temperature and density dependence. Having determined the variation of $\tilde{g}$ with temperature, we can probe the universality of the equation of state close to the quasicondensation crossover. To this aim, we transformed the equation of state by converting $\beta \mu$ into $\beta \mu / \tilde{g}$ and then searched the values of $D_{c}$ and $\mu_{c}$ such that a unique variation emerges for all temperatures studied experimentally.

As shown in Fig. 3, all rescaled equations of state then collapse on a single scaling curve, in striking contrast with the unscaled data displayed in Fig. 1(a). The corresponding values for the critical chemical potential $\mu_{c}$ show a linear dependence on $\tilde{g}$, whereas $D_{c} \approx 8$ remains roughly constant. The latter value also corresponds to the onset of quasi-longrange order in the spatial coherence [11], as well as the temporal coherence discussed below. In contrast to the BKT transition in weakly interacting atomic gases [21], continuously approaching the BEC transition for vanishing $\tilde{g}$ in a harmonic trap [19], for our dipolar gas the transition takes place well above the onset of the Thomas-Fermi region of the density profile where $D \propto \beta \mu$. Also, the saturation of $D_{c}$ confirms a strongly correlated regime away from the dilute gas limit [2]. In the crossover region, i.e., for $D \sim D_{c}$, we actually deduce that the first sound velocity $v_{s}=\sqrt{\hbar^{2} \tilde{g} n} / \mathrm{m}$ is about $10^{4} \mathrm{~m} \mathrm{~s}^{-1}$, i.e., about three orders of magnitude smaller than for exciton polaritons [33]. Therefore, dipolar exciton fluids provide an ideal probe of BKT physics in the hydrodynamic regime dominated by collisions between lowenergy quasiparticle excitations.

We studied the temporal coherence of trapped excitons to independently assess the parameter region of the quasicondensate crossover. Around $D_{c}$ we expect that temporal
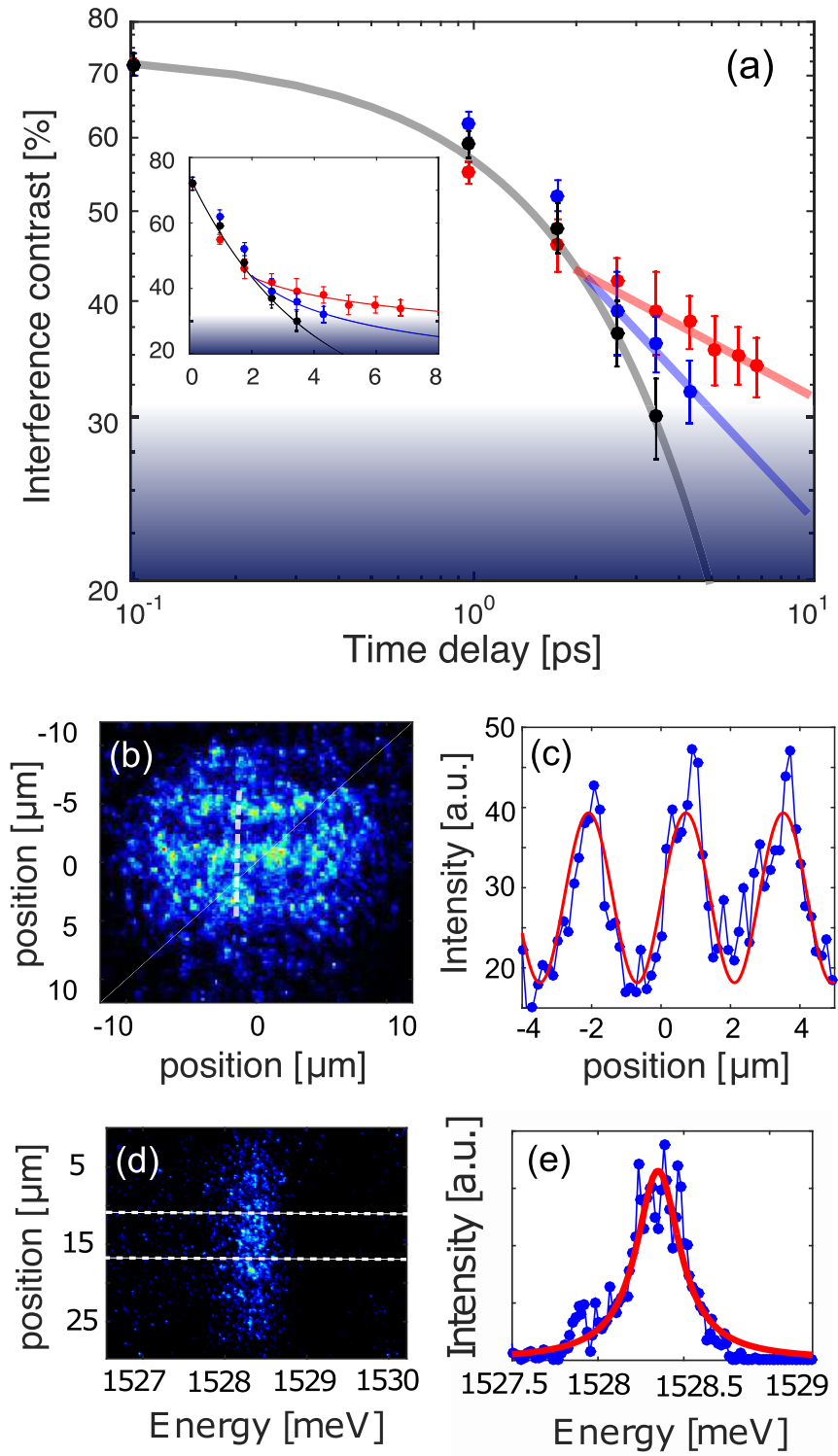

FIG. 4. (a) Amplitude of the first-order temporal coherence $\left|g^{(1)}\right|$ as a function of the time delay for a phase-space density below threshold ( $D=3$ in black), at threshold ( $D \sim 7$ in blue), and above threshold $(D=11$ in red), in log-log scale. The inset displays the same data in linear scale. The black curve shows an exponential decay with a time constant equal to 3.8 ps while the blue and red lines show an algebraic decay $\tau^{-\eta}$ with $\eta=0.4$ and 0.2 , blue and red, respectively, for comparison. The blue shaded area marks the minimum contrast possibly detected for the signal-to-noise ratio of our experiments. (b) Interference pattern measured for $D=11$ and for a time delay set to $5 \mathrm{ps}$. (c) displays the interference signal across the center of the trap [dashed line in (b)], together with a sinusoidal fit to the data leading to a contrast of $35 \%$. (d) Spatially resolved photoluminescence emitted at $340 \mathrm{mK}$ for a phase-space density $D \sim 3$. The spectrum taken at the center of the trap, between the dashed lines, is shown in (e) where the solid red line displays a Lorentzian profile with $320 \mu \mathrm{eV}$ full width at half maximum.

coherence is maintained at long times, thus reflecting directly the building up of spatial order in the system [17,25]. For excitonic systems, temporal coherence is easily accessed, since it is mapped out by the emitted photoluminescence, 
which quantifies the coherence time $\tau_{X}$ of optically active states, i.e., those with an in-plane momentum close to 0 [6]. We then performed time and spatially resolved interferometry in order to quantify $\tau_{X}$, using a Mach-Zehnder interferometer where the photoluminescence field $\psi$ is recombined with itself after a controlled delay $\tau$ is introduced. Figure 4(b) shows the pattern measured above threshold $(D \sim 11)$ for $\tau=5$ ps. Interference fringes are clearly observed all across the trap and we evaluate the interference contrast $\left|g^{(1)}\right|(\tau) \sim$ $\left|\left\langle\psi^{*}(t) \psi(t+\tau)\right\rangle_{t}\right|,\langle\cdots\rangle_{t}$ denoting the average over $t$, by quantifying the modulation amplitude along the vertical axis across the trap [Fig. 4(c)].

Figure 4(a) shows that we observe a clear change of $\left|g^{(1)}\right|(\tau)$ at varying phase-space densities: Below threshold ( $D=3$ in black), the contrast decays exponentially with a characteristic time $\tau_{X} \sim 4 \mathrm{ps}$, which demonstrates that the emitted photoluminescence is homogeneously broadened, with $\sim 300 \mu \mathrm{eV}$ linewidth, as confirmed by direct spectroscopic studies [Figs. 4(d) and 4(e) and the Supplemental Material]. In addition, this exponential decay reflects the twobody collision rate, which is comparable to the value expected in the Thomas-Fermi regime $\Gamma=\hbar \tilde{g}^{2} n / 2 m$. As a result, the interference pattern is not resolved for $\tau$ beyond $1 / \Gamma$. By contrast, around threshold ( $D \sim 7$ in blue), and more markedly above threshold ( $D \sim 11$ in red), the temporal coherence at long delays $\tau$ is maintained. Remarkably, for $D \gtrsim D_{C}$ we note that quasi-long-range order compatible with an algebraic decay $\sim \tau^{-\eta}$ emerges, as clearly seen Fig. 4(a) for data plotted in log-log scale. As is common for systems of mesoscopic sizes, a quantitative determination of the algebraic decay rate will suffer from a considerable systematic bias due to the finite-range limitations. Within our precision, the exponent $\eta$ at criticality is compatible with $\eta_{c} \simeq 0.25$ predicted for a BKT transition in the thermodynamic limit [13]. Averaging the contrast over a finite region with small density variations may result in significantly higher values for the apparent exponent than in strictly homogeneous systems [15,34]. Here, the interference contrast is extracted along one direction in the central density region, making our measurements less sensitive to small density variations, and thus closer to the homogeneous limit.

To conclude, we have reported evidence for a BKT-type transition in exciton fluids with a strong dipolar interaction, by observing a pronounced change from exponential to algebraic decay in the temporal coherence inside a fluctuation region characterized by a universal behavior of the excitonic equation of state. While our measurements of density fluctuations have direct implications for the propagation of first sound, we believe that probing second sound by an additional local heating source, e.g., using an auxiliary laser excitation, is within experimental reach. In the quest for direct probes of superfluid signatures, second sound measurements open the possibility to observe the discontinuous jump at criticality [35], predicted by the BKT theory, which has only been unambiguously observed in helium films [36].

We are grateful to Francois Chiaruttini for important discussions regarding the evaluation of the exciton density, and to Alice Sinatra and Yvan Castin for their crucial help concerning the interpretation of the temporal coherence measurements and helpful comments. We also thank Camille Lagoin for a critical reading. Our work has been financially supported by the Labex Matisse, the Fondation NanoSciences (Grenoble), and by OBELIX from the French Agency for Research (ANR-15-CE30-0020). The work at Princeton University was funded by the Gordon and Betty Moore Foundation through the EPiQS initiative Grant No. GBMF4420, and by the National Science Foundation MRSEC Grant No. DMR 1420541.
[1] C. Trefzger, C. Menotti, B. Capogrosso-Sansone, and M. Lewenstein, J. Phys. B: At. Mol. Opt. Phys. 44, 193001 (2011).

[2] A. Filinov, N. V. Prokof'ev, and M. Bonitz, Phys. Rev. Lett. 105, 070401 (2010).

[3] L. Tanzi, E. Lucioni, F. Fama, J. Catani, A. Fioretti, C. Gabbanini, R. N. Bisset, L. Santos, and G. Modugno, Phys. Rev. Lett. 122, 130405 (2019).

[4] F. Böttcher, J.-N. Schmidt, M. Wenzel, J. Hertkorn, M. Guo, T. Langen, and T. Pfau, Phys. Rev. X 9, 011051 (2019).

[5] L. Chomaz, D. Petter, P. Ilzhöfer, G. Natale, A. Trautmann, C. Politi, G. Durastante, R. M. W. van Bijnen, A. Patscheider, M. Sohmen et al., Phys. Rev. X 9, 021012 (2019).

[6] M. Combescot and S. Y. Shiau, Excitons and Cooper Pairs: Two Composite Bosons in Many-Body Physics (Oxford University Press, Oxford, UK, 2016).

[7] M. Combescot, R. Combescot, and F. Dubin, Rep. Prog. Phys. 80, 066501 (2017).

[8] Y. Mazuz-Harpaz, K. Cohen, M. Leveson, K. West, L. Pfeiffer, M. Khodas, and R. Rapaport, Proc. Natl. Acad. Sci. USA 116, 18328 (2019).
[9] A. A. High, J. R. Leonard, A. T. Hammack, M. M. Fogler, L. V. Butov, A. V. Kavokin, K. L. Campman, and A. C. Gossard, Nature (London) 483, 584 (2012).

[10] R. Anankine, M. Beian, S. Dang, M. Alloing, E. Cambril, K. Merghem, C. G. Carbonell, A. Lemaitre, and F. Dubin, Phys. Rev. Lett. 118, 127402 (2017).

[11] S. Dang, R. Anankine, C. Gomez, A. Lemaitre, M. Holzmann, and F. Dubin, Phys. Rev. Lett. 122, 117402 (2019).

[12] V. L. Berezinskii, Sov. Phys. J. Exp. Theor. Phys. 34, 610 (1972).

[13] J. M. Kosterlitz and D. J. Thouless, J. Phys. C: Solid State Phys. 6, 1181 (1973); J. M. Kosterlitz, ibid. 7, 1046 (1974).

[14] N. D. Mermin and H. Wagner, Phys. Rev. Lett. 17, 1133 (1966).

[15] P. A. Murthy, I. Boettcher, L. Bayha, M. Holzmann, D. Kedar, M. Neidig, M. G. Ries, A. N. Wenz, G. Zurn, and S. Jochim, Phys. Rev. Lett. 115, 010401 (2015).

[16] Z. Hadzibabic, P. Krueger, M. Cheneau, B. Battelier, and J. Dalibard, Nature (London) 441, 1118 (2006). 
[17] D. Caputo, D. Ballarini, G. Dagvadorj, C. S. Muoz, M. De Giorgi, L. Dominici, K. West, L. N. Pfeiffer, G. Gigli, F. P. Laussy et al., Nat. Mater. 17, 145 (2018).

[18] M. Holzmann, M. Chevallier, and W. Krauth, Phys. Rev. A 81, 043622 (2010).

[19] R. J. Fletcher, M. Robert-de-Saint-Vincent, J. Man, N. Navon, R. P. Smith, K. G. H. Viebahn, and Z. Hadzibabic, Phys. Rev. Lett. 114, 255302 (2015).

[20] R. Saint-Jalm, P. C. M. Castilho, E. LeCerf, B. Bakkali-Hassani, J.-L. Ville, S. Nascimbene, J. Beugnon, and J. Dalibard, Phys. Rev. X 9, 021035 (2019).

[21] T. Yefsah, R. Desbuquois, L. Chomaz, K. J. Gunter, and J. Dalibard, Phys. Rev. Lett. 107, 130401 (2011); R. Desbuquois, T. Yefsah, L. Chomaz, C. Weitenberg, L. Corman, S. Nascimbene, and J. Dalibard, ibid. 113, 020404 (2014).

[22] C. L. Hung, X. Zhang, N. Gemelke, and C. Chin, Nature (London) 470, 236 (2011).

[23] N. Prokof'ev and B. Svistunov, Phys. Rev. A 66, 043608 (2002).

[24] D. R. Nelson and J. M. Kosterlitz, Phys. Rev. Lett. 39, 1201 (1977).
[25] N. V. Prokof'ev and B. V. Svistunov, J. Exp. Theor. Phys. 127, 860 (2018).

[26] A. L. Ivanov, E. A. Muljarov, L. Mouchliadis, and R. Zimmermann, Phys. Rev. Lett. 104, 179701 (2010).

[27] C. Schindler and R. Zimmermann, Phys. Rev. B 78, 045313 (2008).

[28] B. Laikhtman and R. Rapaport, Phys. Rev. B 80, 195313 (2009).

[29] V. N. Popov, Functional Integrals in Quantum Field Theory and Statistical Physics (Reidel, Dordrecht, 1983), Chap. 6.

[30] C. Ticknor, Phys. Rev. A 80, 052702 (2009).

[31] See Supplemental Material at http://link.aps.org/supplemental/ 10.1103/PhysRevResearch.2.032013 for additional details regarding the experimental and theoretical procedures.

[32] M. Holzmann, G. Baym, J.-P. Blaizot, and F. Laloe, Proc. Natl. Acad. Sci. USA 104, 1476 (2007).

[33] A. Amo, J. Lefrère, S. Pigeon, C. Adrados, C. Ciuti, I. Carusotto, R. Houdré, E. Giacobino, and A. Bramati, Nat. Phys. 5, 805 (2009).

[34] I. Boettcher and M. Holzmann, Phys. Rev. A 94, 011602(R) (2016).

[35] M. Ota and S. Stringari, Phys. Rev. A 97, 033604 (2018).

[36] D. J. Bishop and J. D. Reppy, Phys. Rev. Lett. 40, 1727 (1978). 